Sah, Y. J., \& Peng, W. (2019). How does interactivity in a video game render people susceptible? A mediating role of concrete-level action identity in immersive media. Cyberpsychology: Journal of Psychosocial Research on Cyberspace, 13(4), article 4. http://dx.doi.org/10.5817/CP2019-4-4

\title{
How Does Interactivity in a Video Game Render People Susceptible? A Mediating Role of Concrete-Level Action Identity in Immersive Media
}

\author{
Young June Sah ${ }^{1} \&$ Wei Peng ${ }^{2}$ \\ ${ }^{1}$ Kangwon National University, Chuncheon, South Korea \\ ${ }^{2}$ Michigan State University, East Lansing, Michigan
}

\begin{abstract}
Action identification theory (Vallacher \& Wegner, 2012) posits that individuals become open to new meanings of actions when the actions are represented in their minds as concrete details (i.e., understanding actions as concrete identities), rather than abstract meanings (i.e., understanding actions as abstract identities). Based on this, we predicted that video game interactivity renders players susceptible to new ideas of actions performed in a video game, by causing them to conceive the actions as concrete details. In a 2 (medium type: interactive vs. noninteractive) $\times 2$ (meaning of hunting: beneficial vs. harmful) between-subjects experiment $(N=111)$, participants played or watched a hunting simulation game, then expressed their agreement on a news article reporting either the benefit or harm of hunting. The interactivity group, compared to the non-interactivity group, described their game experience as concrete actions, indicating that they construed actions as concrete identities. Further, these concrete identities led to greater agreement with the news article regardless whether benefit or harm of hunting was reported. These results suggest an indirect effect of interactivity on participants' susceptibility to a new idea. Implications for using the mental representation approach in media-effect studies were discussed.
\end{abstract}

Keywords: Interactivity; susceptibility; action-identity; mental representation video game

\section{Introduction}

Interactivity has been conceptualized from different perspectives depending on the context in which the concept is employed (e.g., Rafaeli \& Ariel, 2007; Sundar, 2004). Interactivity of immersive media such as video games or virtual realities is defined as a technological characteristic, allowing users to manipulate the form or content of the medium (Steuer, 1992). Previous studies examining interactivity effects in this context often focused on subjective experiences of using an interactive medium (i.e., sense of being in a mediated or artificial environment, or presence, Sanchez-Vives \& Slater, 2005; identifying themselves with a video game character, or character identification, Lin, 2013), considering subjective experiences as immediate psychological effects of an interactive medium and as mediators to further examine effects of interactivity (e.g., hedonic effects of avatar connection, Klimmt, Hefner, \& Vorderer, 2009; persuasive effects of social presence, Skalski \& Tamborini, 2007).

While these studies relied on subjective-level psychological constructs as mediators for interactivity effects, they paid less attention to cognitive aspects of the interactivity effect, that is, how interactivity influences the way people mentally represent their experiences in the medium, and what consequences the different ways of forming mental representation have. Mental representation has been a useful tool in psychology and media-effect studies in understanding a wide range of cognitive processes (Carlston \& Smith, 1996), and media-related constructs, e.g., social-reality construction (Shrum, 2009). 
The current study adopted mental representation as a key construct in explaining the mechanism of psychological effects of interactive media. Particularly, the current study predicted that interactivity in a video game increases persuasive effects of the medium, because interactivity facilitates information processing (i.e., the elaboration likelihood model, Petty \& Briñol, 2012), specifically information regarding how actions are performed in the video game, and causes users to form more concrete mental representations of actions. Our hypothesis was guided by the action identification theory (Vallacher \& Wegner, 2012), which proposes that individuals become susceptible to a new meaning of an action when they represent the action as concrete details in their minds (i.e., concrete identities) rather than abstract meanings of the action (i.e., abstract identities). Based on this theory, we predicted that interactive media, compared to non-interactive, may cause actions performed in the media to be presented as more concrete-level identities and, in turn, render users susceptible to a new meaning of the actions.

While media-effect studies employing the mental representation approach mainly relied on accessibility (RoskosEwoldsen, Roskos-Ewoldsen, \& Carpentier, 2009), not many studies have focused on the concreteness of the mental representation. Some recent studies examined the concreteness of mental representation in the video game context, but their investigations focused on the role of individuals' prior game experiences (Ewell, Hamilton, \& Guadagno, 2018; Matthews, 2015). In contrast, our current study attempts to utilize the construct of concreteness in investigating the interactivity effect on persuasion. We suggest that the concrete action identity has potentials not only for extending our understanding of psychological effects of media technologies, but also for presenting implications regarding utilization of media technologies in different domains. As media technologies aim to provide realistic thus concrete experience of mediated and virtual environments (e.g., virtual reality), showing that the level of concreteness mediates the persuasive effect does present a theoretical justification for utilizing such technologies for desired outcomes (e.g., changing beliefs on health-related topics).

\section{How Do People Represent Action in Their Minds?}

According to the action identification theory (Vallacher \& Wegner, 2012), individuals produce mental representations of actions with different levels of abstraction, depending on situations in which they perform the actions (Burgoon, Henderson, \& Markman, 2013). The level of abstraction is associated with aspects of actions on which the individuals focus (i.e., action identity). When construed at a concrete level, actions are identified as perceptual details such as specific body movements and kinetic sequences, all of which are devoted to defining how actions are executed. Construed at an abstract level, actions are understood in terms of high-level thoughts, such as goals, meanings, and values of actions, indicating why an action is performed.

While people identify an action with different natures at a low- or high-level of abstraction depending on personal traits (Vallacher \& Wegner, 1989) or situational factors (Trope \& Liberman, 2010), they usually construct their action identities at a high level for the cognitive benefit of abstract identities. That is, action identities at the abstract level allow people to conceive an action in a more efficient and comprehensive manner than do identities at the concrete level, thus enable people to focus on their original intentions of performing actions. However, when people fail to execute the action successfully with abstract identities they perform the action with concrete identities, as concrete identities are informative about how to perform actions (Vallacher \& Wegner, 2012). Not until they have mastered the concrete-level actions do they shift their action identity to the abstract level for comprehensive maintenance.

To illustrate, consider the example of toasting bread. If construed abstract, the action can be represented as a high-level goal of the action, e.g., preparing breakfast; if construed concrete, the action can be represented as a sequence of specific motions, e.g., operating a toaster. The concrete identity offers information regarding the means to toast bread, whereas the abstract identity informs why the bread is being toasted. During the course of the action, people usually think of the behavior as preparing breakfast (i.e., an abstract identity) as it is the immediate purpose of the action. When the actor is confronted with a problem in the action and cannot perform smoothly the action with reference to the abstract-level identity (e.g., a jammed lever), the concrete-level identity of operating a toaster replaces the high-level meaning of breakfast preparation. After resolving the problem, the actor may discontinue attention to the concrete-level identity and again think of it in terms of its abstract-level purpose. As such, action identification is a dynamic process, through which actors develop mental representations of their actions adequate for situational requirements. 
Vallacher and Wegner (2012) further posited that representing an action at a concrete level renders people susceptible to cues denoting a high-level meaning of the action. Called the emergence process (Vallacher \& Wegner, 2012), this notion suggests that people are likely to change their high-level understanding of an action and embrace new abstract-level identities only when they perform actions with concrete-level identities. In contrast, when actions are represented at an abstract level and people have developed their own understanding of those actions, they hardly accept another abstract meaning of the action and refuse to change their ways of understanding, given their preoccupation with what the actions mean to them.

Empirical studies have documented evidence supporting susceptibility to new meanings engendered by concretelevel action identities (Ledgerwood, Trope, \& Chaiken, 2010). For example, Wegner, Vallacher, Macomber, Wood, and Arps (1984) tested whether thinking of an action at a concrete level causes people to agree with a different abstract-level idea of the action. One group of participants was asked to list actions regarding "going to college" at a concrete level (e.g., "walking around campus"), and another group was asked to list actions at an abstract level (e.g., "improving my chances of getting a job I want") to manipulate their identity levels of the action, going to college. They then were exposed to a new meaning related to the action by reading one of two fabricated news articles reporting an effect of college education on their sex lives ("improving one's sex life" or "impairing one's sex life"). Confirming researchers' predictions, the group thinking of the action as concrete-level identities reported greater agreement with the alleged effect in the news article than did the group thinking of the action as abstractlevel identities, regardless whether the articles stated that college education improved or impaired one's sex life. This result suggests that those with more concrete-level identities are likelier to accept an unthought meaning of "going to college," than are those with abstract-level identities.

\section{How Do Interactive Media Render People Susceptible?}

The emergent process of action identification theory suggests that an interactive medium could render people receptive to a new idea about an action, by facilitating a concrete-level action identity performed in the medium. Research on virtual reality and cognitive science supports this prediction, as we discuss below.

Action identity of interactive media. To the best of our knowledge, no direct evidence has been reported in media-effect literature regarding how interactivity influences action identity. However, some studies in virtual reality offer indirect evidence. Studies on motion training (Bailenson et al., 2008) and rehabilitation (Schultheis \& Rizzo, 2001) showed interactive virtual reality's advantage over non-interactive media in the area of physical motion training, implying that interactivity may promote the process of perceptual information of motion, at a concrete level. Bailenson et al. (2008) reported that an interactive virtual reality, as compared to a pre-recorded video, enhanced effectiveness of the medium in training physical motions, because interactive virtual reality allowed participants to pay attention to detailed configurations of actions portrayed on screen. Note that attending to detailed motion configuration means developing concrete-level representation in one's mind (Vallacher \& Wegner, 2012). Thus, this result suggests that an interactive virtual reality tends to prompt users to form more concrete than abstract identities of motions.

Literature on self-agency also indicates that using interactive media may foster concrete-level action identity. When using interactive media, people acknowledge they are causing the action presented in the media by observing the avatar's movements and/or changes in the environment portrayed in the media. Perceiving selfgenerated actions is different from perceiving other-generated actions (Blakemore \& Frith, 2003), as only the former entails a sense of agency, i.e., a sense that one is the generator of the action (van der Weiden, Aarts, \& Ruys, 2013). This sense of self-agency is central to experiencing interactivity of media. When people perform actions in an interactive medium, they expect sensory feedback corresponding to their inputs. If sensory feedback matches their expectations of their inputs, they feel they brought about changes in the media. Considering that sensory feedback entails concrete information of actions rather than abstract meanings, expectation of feedback in selfagency would result in representing an action as a concrete identity.

Action identity in non-interactive media. Regarding mental representations formed from actions displayed in non-interactive media, literature on social cognition offers scant yet meaningful clues. In particular, studies on goal inference suggest that humans in unconscious and unintentional ways can infer abstract mental states from others, such as trait inference (Uleman, Newman, \& Moskowitz, 1996) and goal inference (Aarts, Gollwitzer, \& 
Hassin, 2004). This tendency is so pronounced that people infer mental states even from non-human instances (Heider \& Simmel, 1944). This line of literature indicates that people are likely to extract high-level meanings from their perceptions, and unlikely to retain details of visual stimuli.

Our prediction that non-interactive media are less likely to lead to concrete action identities is based on the fact that non-interactive media such as movies or television shows typically present understandable stories or meaningful behaviors of characters. When visual stimuli present a meaningful story, an audience would be less likely to represent characters' actions with their specific bodily motions, yet more likely with meanings relative to a broader context. Supporting this notion, evidence shows that people tend to harness a narrative to understand causal relationships among social events, and to predict future events (Costabile \& Klein, 2008). As inferring causality and predicting events require one to abstract social meanings from characters' behaviors and surrounding contexts, the tendency to follow a narrative indicates that people tend to form more abstract-level action identities than to encode specific actions of characters when watching non-interactive media.

These lines of argument for the effect of interactive and non-interactive media on the level of action identity lead us to our H1, stated so:

H1: Compared to those using a non-interactive medium, people using an interactive medium are more likely to represent actions as concrete-level identities.

As action identification theory suggests, the level of action identity influences susceptibility to a new idea of an action, such that a concrete-level action identity renders people susceptible to a new idea. Based on this argument, our $\mathrm{H} 2$ predicts:

H2: The more people represent their actions as concrete-level identities, the more they become susceptible to new ideas of those actions.

These two hypotheses lead us to the prediction that the concrete-level of action identity mediates the effect of interactivity on susceptibility. Thus, our H3 is stated so:

H3: Concrete-level action identities mediate the effect of interactivity, such that interactivity, compared to non-interactivity, leads to a greater level of concrete-level action identities and, in turn, concrete-level identities render people susceptible to new ideas of the actions.

\section{Method}

\section{Overview}

To test our hypotheses, we conducted an online study and a main study. The online study was undertaken to garner Likert-type items for measuring concrete-level identities, and to pretest materials used in the main study to measure susceptibility to a new meaning of hunting. In the main study, participants played (or watched) a hunting simulation game and reported their experiences of using the medium, by which we assessed the extent to which participants formed concrete identities of actions involved in hunting. Following Wegner et al. (1984), susceptibility to a new idea was assessed in a purportedly-unrelated task, in which participants read a news article reporting effects of hunting, and rated the extent to which they agreed with it.

\section{Online Study for Item Development}

We conducted an online study to develop items measuring action identities. College students $(n=96)$ at a large university in the Midwest of the United States participated in the online study. They were recruited using a collegeoperating online recruitment system from September 8 to 16, 2014. We used a thought-listing technique to collect items for the action identity measure, through which participants were asked to think for 30 seconds about hunting and describe their thoughts. Half were randomly selected for the concrete-identity condition and instructed to imagine hunting in terms of specific actions and bodily motions involved in deer hunting (how-to-hunt 
items). Considering concrete-level action identities indicate specific motions involved in hunting deer, items regarding how to hunt are appropriate for the measurement of the concrete-level identity. The other half were assigned to the abstract-identity condition and asked to think broadly about motives for and consequences of hunting (why-to-hunt items). Following that, all participants were given one minute to list six thoughts that came to mind. After reviewing the responses, we selected eighteen thoughts mentioned most frequently--nine each from the how- and the why-to-hunt thoughts (see Appendix A).

Participants then moved to the second part designed to pretest news articles. We crafted two news articles with different meanings of deer hunting, one declaring harmful effects (i.e., "hunting increases risk of heart disease"), the other reporting beneficial effects (i.e., "hunting enhances cognitive ability," see Appendix B). While we designed these news articles to present an unthought meaning of hunting for participants in the main study, we wanted them to be moderately believable. The news articles were tested by participants randomly assigned to one of the two versions. They then reported their agreements using six items (see Appendix A) on a 7-point scale ranging from 1 (strongly disagree) to 7 (strongly agree). Ratings were averaged to generate a composite measure, which showed a moderate level of agreement, $a=.90, M=4.80, S D=1.12$, greater than the mid-point $4.0, t(95)=7.00, p$ $<.001$.

A 2 (action-identity level: abstract vs. concrete) $\times 2$ (meaning of hunting: hunting is beneficial vs. harmful) ANOVA revealed that agreement on the two news articles did not differ, $F(1,92)=0.35, p=.556$ (for the harmful effect, $M$ $=4.71, S E=0.14$; and for the beneficial effect, $M=4.85, S E=0.19$ ). However, the action-identity level influenced the agreement level, $F(1,92)=4.92, p=.029, \eta_{p}^{2}=.10$, such that those who conceived concrete-level identities were more likely to agree with the news article $(M=4.84, S D=1.04)$ than did those who had abstract-level identities $(M$ $=4.42, S D=1.12$ ). This result lent support to the notion that concrete action identities lead to greater susceptibility to a new idea. The action-identity level by meaning of hunting interaction was not significant, $F(1,92)=1.91, p=$ .170 .

\section{Main Study}

Design and participants. Data were collected from a 2 (medium type: interactivity vs. non-interactivity) $\times 2$ (meaning of hunting: hunting is beneficial vs. harmful) between-subjects experiment. With our main research purpose being to examine effects of interactivity on individuals' susceptibility to a new idea, we employed two plausible meanings of hunting-i.e., hunting is a beneficial activity and hunting is a harmful activity -as a second between-subjects factor, to generalize our findings. Participants $(N=111$; male $=39$, female $=72)$ were recruited using the online recruitment system from October 15 to November 13, 2014. A recruitment for a study posted on the system informed participants that they would be asked to play a video game and report their experience. Participants' age ranged from 18 to $27(M=20.70, S D=1.45)$. Participants were randomly assigned to one of the experiment groups. None of them dropped the experiment. Table 1 shows that participants' age and gender across the experiment groups. Note that the participants' gender was not evenly distributed across the conditions, indicating that the effect of interactivity may be confounded with the gender effect.

Table 1. The Number of Male and Female Participants and Average Age (Standard Deviation) Across Experiment Groups.

\begin{tabular}{lcccc}
\hline & \multicolumn{2}{c}{ Non-interactive condition } & \multicolumn{2}{c}{ Interactive condition } \\
\hline Beneficial condition & Female $=21$, Male $=9$ & $M_{\text {age }}=20.37(1.30)$ & Female $=22$, Male $=3$ & $M_{\text {age }}=20.28(1.10)$ \\
Harmful condition & Female $=16$, Male $=10$ & $M_{\text {age }}=20.92(1.29)$ & Female $=13$, Male $=17$ & $M_{\text {age }}=21.20(1.29)$ \\
\hline
\end{tabular}

Materials. The experiment used the video game The Hunter (Avalanche Studios, 2009), a first-person-perspective hunting simulation game (see Figure 1), in which a player sees the environment through an avatar's eyes. We utilized two simple missions of the video game in the experiment. The first was for players to learn basic controls of moving and firing a rifle. Guided by instructions, participants climbed a tower from the top of which they aimed at and shot a deer. The second asked players to locate deer by monitoring a GPS device and tracking footprints. Once arriving at a hunting site, participants used a deer caller to attract deer, and rifle-shot them as they approached. The video game was displayed on a 27-inch monitor with a $2560 \times 1440$ pixel resolution. 
Interactivity vs. non-interactivity. The interactivity group played the two missions with mouse and keyboard. They controlled the avatar's movements using the keyboard, and maneuvered direction and rifle-aim using the mouse. The non-interactivity group watched a video clip prepared by recording the two missions played by a confederate with no previous experience of the game.

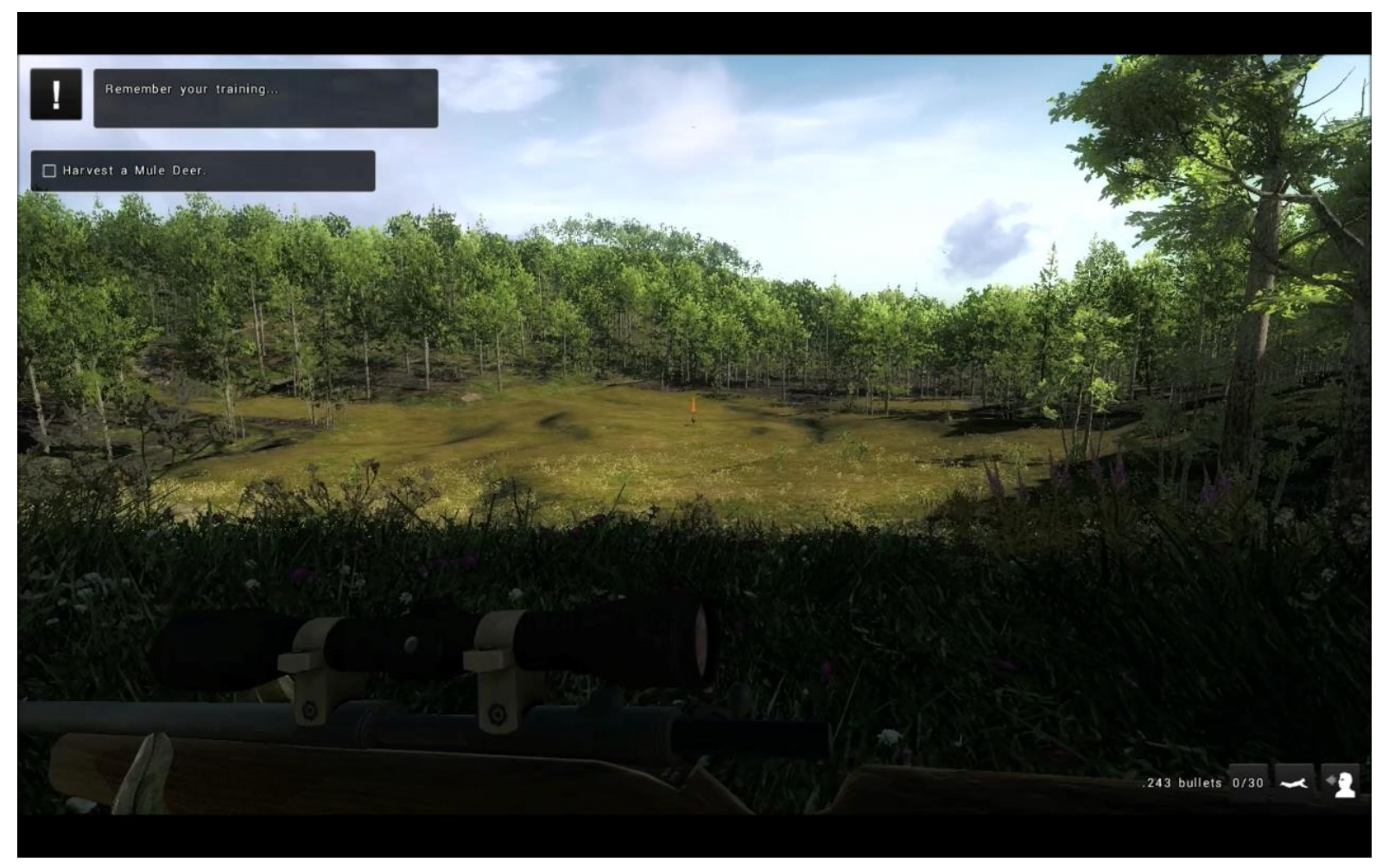

Figure 1. A captured image of The Hunter (Avalanche Studios, 2009).

Measures. As mental representations are developed unconsciously (Banaji, Lemm, \& Carpenter, 2001), direct asking can be an inappropriate way to measure the construct (Fazio \& Olson, 2003). Previous studies instead employed indirect ways (see Burgoon et al., 2013). We employed both direct and indirect approaches in a postgame questionnaire: a concrete identity score, based on self-reporting on Likert-type items; and a linguistic category score, developed on the thought-listing technique. Also, our dependent variable, agreement with the news article, is measured in a direct way, as it pertains to one's conscious judgment on a certain issue.

Mediators. The concrete identity score was calculated using Likert-type items collected from the online study. Participants were asked to rate how well the eighteen items described their experience on a 5-point scale ranging from 1 (very poorly) to 5 (very well). The order of items was randomized for each participant. Participants' ratings were analyzed using exploratory factor analysis with the promax rotation. A parallel analysis suggested two factors, collectively explaining $50.08 \%$ of the variance, and items with mid- to high-loadings $(>.60)$ were retained in the measure. The first factor included six from the why-to-hunt items, and the second factor five from the howto-hunt items (see Appendix A). Having our focus mainly on the concrete-level action identities, we employed only the concrete-level identity. Participants' ratings on the five items in the second factor were averaged to generate the concrete identity score, $\alpha=.88, M=3.74, S D=0.89$.

The Linguistic category score was constructed by quantifying the concreteness level of responses obtained from a thought-listing item measured in the post-game questionnaire. The thought-listing item presented participants with six blanks and asked them to describe their experience in the hunting simulation game. Participants offered short descriptions up to six times, using a word (e.g., "chasing") or a few (e.g., "aiming a gun at the deer"). Each participant's average number of words for the six responses ranged from 1.0 to 11.5 , with the grand average for all participants being $2.97(S D=3.20)$.

Participant's responses were quantified using a coding scheme (see Appendix C), developed by adjusting the Linguistic Category Model (LCM; Semin \& Fiedler, 1991). Semin and Fiedler (1991) proposed that individuals form mental representations of other people at different levels of abstraction, and that abstraction levels can be 
assessed by examining their language used in describing others. LCM assumes that individuals tend to use verbs denoting specific actions for concrete mental representations, whereas they employ adjectives for abstract representations as adjectives imply generalized traits (Semin \& Fiedler, 1988, 1991). Harnessing this idea, we assessed the level of concreteness in participants' descriptions of their in-game experiences. First, we classified a subject of each response into either participant or game, by identifying whether the response refers to participants' own experience (e.g., "excitement" or "shooting deer"), or video game contents (e.g., "a deer caller"). For responses coded as participant subject, we further assessed their abstraction level by applying the five categories adopted from LCM--Descriptive Action Verb (DAV), Interpretive Action Verb (IAV), State Action Verb (SAV), State Verb (SV), and Adjective (ADJ). DAV, being the most concrete category, refers to a concrete and graphic description of body movements. Responses denoting a specific motion performed in the course of hunting (e.g., "crawling") were coded into DAV. IAV is more abstract than DAV, as IAV refers to an action that goes beyond specific body movements to contextual meanings of action. Because it is not associated with a specific body motion, IAV can be done via different means. Responses referring to an action executed by different specific motions (e.g., "moving") were coded into IAV. SAV refers to a verb expressing psychological states caused by an action. Responses indicating emotional and cognitive states caused by a particular event (e.g., "felt nervous,") were coded into SAV. SV also refers to a mental status, but without any clear causes associated with the state. It is more abstract than SAV, as SV does not assume a particular event causing the state, but simply refers to an enduring state. Responses indicating a mental state without any clear causes were coded into SV. LCM also includes ADJ, the most abstract category, which denotes stable attributes of objects of interest. We applied this category to responses referring to an attribute of participants (e.g., "being stealthy").

Participants' responses $(N=626)$ were coded by two independent coders, blind to conditions. They achieved acceptable agreement, Cohen's K $=0.76$ (Landis \& Koch, 1977). Disagreements were resolved by discussion based on the codebook. Coded responses were used to create the linguistic category score, following the LCM (Semin \& Fiedler, 1989). The LCM assigned higher points to a less concrete category: four points for ADJ, three for SV, two for SAV and IAV, one for DAV. The linguistic category score for a participant was calculated by dividing the sum of the points for the participant's responses by the number of his/her responses, $M=1.72, S D=0.44$. The lower a score was calculated, the more concrete action identities it assumed she/he developed. For example, a participant, who answered with four DAVs and two IAV, thus scored 1.33 ( = (4X1+2X 2) / 6), constructed more concrete action identities than did a participant who answered with three ADJs and three SAVs and recorded $3.00(=(3 \times 4$ $+3 \times 2)$ / 6). Participants who offered no responses to the thought-listing item $(n=4)$ were excluded when the linguistic category score was analyzed.

Dependent variable. Agreement with the news article was used as our dependent variable, measured by the six items used in the pilot study on a 5-point Likert scale with higher score indicating greater agreement (Appendix A). Ratings on the items were averaged for each participant for an index of agreement ( $\alpha=.87, M=4.61, S D=1.11$ ).

Procedure. On arrival at the experimental laboratory, participants reviewed experiment procedures and indicated their consents. They then were randomly assigned to one of the medium-type conditions. For approximately 15 minutes, the interactivity group played the game and the non-interactivity group watched the pre-recorded video. Next, participants were instructed to rest 5 minutes before completing the post-game questionnaire. During this resting period, a confederate asked them to participate in a simple pilot study. They were told that the study was a pilot study of a different project. All agreed to participate. In the ostensible pilot study, participants were given a news article, reporting either beneficial or harmful effects of hunting. Using this cover story, we tried to hide the relevance between the pilot study and the main study, as awareness of the relevance may cause participants to correct their responses (Förster \& Liberman, 2007). Selection of the news article was determined randomly, and researchers were blind to this condition. Participants read the article and evaluated to what extent they agreed with the news article. Participants then resumed the original study, completing a post-game questionnaire on a different computer. In the questionnaire, participants responded to the open-ended question item for the linguistic category score, then to the Likert-type items for the concrete identity score. At the end of the study, participants were debriefed on the intent of the experiment and the relevance of the ostensible pilot study. Prior to leaving, all participants were asked whether they were aware of the relevance of the pilot study; none reported being so. 


\section{Results}

\section{Preliminary Analysis for Thought-Listing Responses}

A frequency analysis showed that words presented for the thought-listing item differed between the interactive and non-interactive condition, such that nouns (i.e., deer and animal) appeared less often, and verbs denoting specific actions (i.e., shooting and tracking) more often in the interactive condition than in the non-interactive condition (see Table 2).

Table 2. Frequency Distribution of Words Appearing in the Thought-Listing Task.

\begin{tabular}{|c|c|c|c|c|}
\hline \multirow{2}{*}{ Rank } & \multicolumn{2}{|c|}{ Non-interactive condition } & \multicolumn{2}{|c|}{ Interactive condition } \\
\hline & Word & Frequency & Word & Frequency \\
\hline 1 & deer & 61 & deer & 47 \\
\hline 2 & shooting & 21 & shooting & 29 \\
\hline 3 & hunting & 17 & tracking & 19 \\
\hline 4 & animal & 16 & hunting & 14 \\
\hline 5 & tracking & 14 & using & 13 \\
\hline 6 & killing & 12 & killing & 11 \\
\hline 7 & felt & 10 & aim & 9 \\
\hline 8 & find & 9 & animal & 9 \\
\hline 9 & target & 8 & excitement & 9 \\
\hline 10 & gun & 8 & gun & 8 \\
\hline
\end{tabular}

Note. Only nouns and verbs counted.

Table 3 shows the results of coding participants' responses to the thought-listing item. A large number of responses described participants' own experiences (77.7\%) rather than video game contents and a majority of participants' experiences (97.1\%) were DAV or IAV or SAV. Note that none of the responses were categorized into $\mathrm{SV}$, indicating that all responses in the verb form referred to temporal experience gained from the video game. A chi-squared test revealed that the numbers of responses in the categories were dependent on the medium-type condition, $\chi^{2}(4)=41.01, p<.001$.

Table 3. Number of Responses Coded into Each Category.

\begin{tabular}{lcccccc}
\hline \multirow{2}{*}{ Medium type } & \multicolumn{4}{c}{ Participants-subject responses } & Game-subject \\
\cline { 2 - 6 } & DAV & IAV & SAV & SV & ADJ & responses \\
\hline Non-interactive & 53 & 52 & 114 & 0 & 6 & 77 \\
Interactive & 124 & 24 & 102 & 0 & 8 & 62 \\
\hline
\end{tabular}

\section{Hypothesis Test}

In our initial analyses, we tested hypotheses with potential covariates such as previous hunting experience ( $14.41 \%$ of the participants had hunting experience), and gender (64.86\% female, $35.14 \%$ male), but none were found to change the results of hypothesis testing. Thus, we reported the results of parsimonious analyses here excluding potential covariates.

$\mathrm{H} 1$ was tested using a series of 2 (medium type) $\times 2$ (meaning of hunting) ANOVA. While we did not expect any effect of meaning of hunting, we included it in the model to generalize our results. Using the concrete-identity score as a dependent variable, results indicated no evidence for the main effect of medium type, $F(1,107)=1.41$ $p=.238$, nor for meaning of hunting, $F(1,107)=0.08, p=.781$. The medium type by meaning of hunting interaction was not significant also, $F(1,107)=0.13, p=.720$. 
Using the linguistic category score as a dependent variable, results showed that medium type had a significant main effect, $F(1,103)=4.87, p=.029, \eta_{p}{ }^{2}=.05$. Supporting $\mathrm{H} 1$, the linguistic category score for the interactivity group $M=1.63, S E=0.06$ was higher than the score for the non-interactivity group, $M=1.82, S E=0.06 ; t(103)=-$ $2.21, p=.029$. The main effect of meaning of hunting, $F(1,103)=0.02, p=.678$, and the interaction term, $F<0.13$, $p=.722$, were not significant. Thus, our analysis lent mixed support for $\mathrm{H} 1$.

$\mathrm{H} 2$ was tested using an OLS regression. Results showed that the concrete-identity score was not associated with agreement, $b=0.18, S E=0.12, t=1.49, p=.104 ; R^{2}=.02, F(1,109)=2.22, p=.139$. When the linguistic category score was used as a predictor, it did predict agreement, $b=-0.58, S E=0.25, t=-2.33, p=.024 ; R^{2}=.05, F(1,105)=$ $5.43, p=.022$. Considering that the linguistic category score was a reversed measure for the concrete identity, this result implies that participants with greater concrete identity were likelier to agree with the news article. Thus, our analysis provided mixed support for $\mathrm{H} 2$.

As our results showed that the interactivity group revealed a lower linguistic category score $(\mathrm{H} 1)$ and that a low linguistic category score was associated with greater agreement $(\mathrm{H} 2)$, it is possible that interactivity indirectly influences agreement on a news article (H3). Using the bootstrap method in the package mediation in R (Tingley, Yamamoto, Hirose, Keele, \& Imai, 2014), we conducted a mediational analysis and estimated the indirect effect of interactivity based on 5000 bootstrap samples. A significant indirect effect of interactivity on the agreement was found, $b=.12, p=.032,95 \% \mathrm{Cl}[.01, .29]$, while the direct and the total effects were not significant, $b=-.25, p=$ $.232,95 \% \mathrm{Cl}[-.69, .16]$ and $b=-.14, p=.532,95 \% \mathrm{Cl}[-.57, .29]$, respectively. Thus, this result lent support to $\mathrm{H} 3$. Non-significant total effect was also confirmed in a 2 (medium type) $\times 2$ (meaning of hunting) ANOVA, for medium interactivity, $F(1,107)=0.34, p=.563$, and meaning of hunting, $F(1,107)=0.16, p=.687$. The interaction was not significant, $F(1,107)=0.11, p=.747$. Thus, the result did not reveal evidence for the overall effect of interactivity on susceptibility.

\section{Discussion}

Applying the mental representation approach to media-effect research, the current study proposed that interactivity led to more concrete-level identities for activities performed in a video game $(\mathrm{H} 1)$ and that concrete action identities rendered individuals susceptible to new meanings of the activities $(\mathrm{H} 2)$. Therefore, interactivity generates the persuasive effect mediated by concrete action identities (H3). Part of our data collected in the current study rendered evidence for these hypotheses, particularly for those collected using a thought-listing technique. Responses to the open-ended question in the thought-listing technique showed that the interactivity group, compared to the non-interactivity group, presented responses more in concrete-level categories, lending support to H1. Particularly, the interactivity group offered a larger number of responses in DAV than IAV, whereas the non-interactivity group presented similar numbers to the two categories. The interactivity group, being generators of actions in the medium, was likely to conceive their actions as kinetic sequences of movements, and to describe their experiences with terms denoting specific bodily movements. In contrast, the non-interactivity group, being observers of actions, tended to be inattentive to - thus did not bear in mind - detailed configurations of the game character's actions. Rather, the non-interactivity group focused on interpretational aspects of actions (i.e., IAV), thus used fewer concrete terms indicating specific bodily actions.

Also, results from the current study yield partial support for $\mathrm{H} 2$, replicating the emergence process of concrete action identity (Wegner, et al., 1984) in a video game context. As evidenced in the negative relationship between the linguistic category score, and agreement with the news article, the more concrete-level terms participants used and the less abstract-level terms, the more likely they were to agree with an unthought but likable consequence of hunting. This finding implies that those who experienced their game play as concrete-level actions are likelier to become susceptible to a new meaning of actions they performed in the game.

Further, $\mathrm{H} 3$ predicted an indirect effect of interactivity on susceptibility, via the concrete action identities. Our results showed that the linguistic category score had a mediational role for the weak but significant indirect effect of interactivity, implying that the persuasive effect of interactivity is produced by causing media users to form concrete mental representations of their media experience. Despite the absence of the total effect of interactivity on susceptibility, an indirect effect can exist, particularly when other mediators suppress the effects of interest (Hayes, 2009). 
One possible explanation for the non-significant total effect might be that the peculiarity of this experiment's noninteractive condition gave participants a concrete-level experience. That is, because the video game used for the current experiment was biased in describing specific actions involved in hunting deer, it did not generate enough variations in the action identities of participants' experiences. Specific tasks of the video game we used in the experiments displayed written instructions on how to control a game character whenever the character needed to perform actions. We believe these instructions may have yielded concrete action-identities, and increased susceptibility for both interactivity and non-interactivity groups as the instructions informed users how to control a game character. Thus, the total effect generated by the interactivity condition is not large enough to be detected in our analysis. This result implies that non-interactive media also can cause media users to develop concrete action-identities, particularly when those users are guided to focus on actions rather than meanings and implications of the actions portrayed on the media. However, interactive media by nature tend to facilitate the development of concrete action identities, as indicated by our result on the thought-listing item, and we captured the effect only when we examined it being mediated by concrete action-identities.

This reasoning is in line with our comparison between high-level action-identity conditions in the online study, and in the main study. That is, the non-interactive group's agreement with the news article in the main study was marginally higher $(M=4.79, S D=1.16)$, than that of the why-to-hunt group in the online study $(M=4.42, S D=1.12$, $t(146)=1.73, p=.085)$. Given that the agreement positively relates to the concrete action-identity, this analysis suggests that the non-interactivity group of the main study, who watched the video game, experienced the contents as concrete action identities to a greater extent than did the why-to-hunt group of the online study, who imagined deer hunting as goals and meanings. Therefore, we suspect that inflated concrete-level identities in the non-interactive condition resulted in the non-significant total effect of interactivity on susceptibility.

\section{Implications}

The current study contributes to media-effect research from different aspects, presenting theoretical, methodological, and practical implications. Theoretically, this study introduced to media-effect research an underexplored mediator, concrete-level action identification, and expanded our knowledge of the underlying mechanism of how media interactivity promotes the persuasive influence of a post-media message. Note that our findings are not against previous studies examining reinforcing effects of interactivity, which have focused on perceptual (e.g., Steuer, 1992; Wu, 2005) or cognitive dimensions and found attitudinal effects of interactivity (Oh \& Sundar, 2015). In general, previous studies indicate that interactivity encourages media users' information processing (e.g., systematic information processing in the elaboration likelihood model, Petty \& Briñol, 2012), either by allowing them to be immersed in media (i.e., presence, Steuer, 1992 or cognitive absorption, Oh \& Sundar, 2015) or by facilitating them to assimilate a character's internal state (i.e., character identification, Lin, 2013). In contrast to these studies focusing on subjective experiences of media use, our current study sheds lights on another side of the cognitive process, namely the level of concreteness, regarding how interactivity of immersive media induces persuasive effect.

We believe that the introduction of the novel mediator deepens our understanding of the media-effect mechanism, as it specifies the cognitive characteristics of the enhanced information processing. Immersion in media or identification with a character may involve processing a greater amount of information presented by a medium, as suggested in previous studies (Lin, 2013; Steuer, 1992). This information processing may incorporate individuals' development of concrete-level media experience, and such concrete action identities, which, in turn, may lead to reinforcing persuasive effects of media contents by promoting adoption of a new high-level meaning of media contents. Thus, this specification of the process suggests the boundary condition of the media effect generated by the enhanced information processing: The enhanced information process engendered by media consumption would foster the persuasive effect, particularly when media consumption enables users to construe concrete identities of media contents.

Further, the mediator examined in the current study can be used to understand media effects in a broader context. While we used the level of concreteness as a mediator for the interactivity effect, the construct can be used to examine also the effects of non-interactive media. Previous studies on the effect of non-interactive media acknowledge that concrete aspects of media contents (e.g., narrative) can release their audience from an existing high-level idea (e.g., ideology), causing them to reshape their conceptions (Slater, Rouner, \& Long, 2006). In 
particular, studies drawing on exemplification theory (Zillmann, 2006) have accumulated evidence for persuasive effects of a concrete exemplar in non-interactive media, in comparison to its abstract counterpart of quantitative information (Westerman, Spence, \& Lin, 2015; Zillmann, Gibson, Sundar, \& Perkins, 1996). While several mechanisms have been proposed (Zillmann, 2006), the current study presented an additional layer to the mechanism: An exemplar may facilitate construction of concrete-level mental representations of messages, rendering media users more susceptible to new messages thereafter. By addressing subtle psychological processes of media use, the current study thus complements existing literature on pronounced media effects, such as formation or modification of beliefs (Zillmann, 2006).

When applied to the aggressive effects of violent video games, using the action identity level as mediator gives additional insight into the existing literature. According to the action identification theory (Vallacher \& Wegner, 2012), novice video game players are expected to focus on perceptual details of video game contents and construct concrete action identities, because of the unfamiliarity of the video game contents and controls. For novice players, behaviors involving physical force to hurt or kill someone in a video game can be interpreted as using violence, a high-level identity inferable immediately from the contents. When this concrete-level identification generates assimilating effects, players would reveal aggressive affect, aggressive cognition, and aggressive behaviors, along with physiological arousal, as evidenced in the relevant literature (Anderson, 2004). For experienced players, however, violent actions performed in the video game is associated with a high-level meaning other than aggressive behaviors, which is often embedded in its story line of the video game (e.g., defeating enemy or protecting their nation) or developed by players' own sense-making process (e.g., demonstrating their skills). This high-level identification of violent behaviors may also result in aggressive outcomes, but in a different mechanism, such as desensitization to violence or decreased empathy for a victim of violence (Bartholow, Sestir, \& Davis, 2005). Thus, action identification introduces testable predictions with distinctive mechanisms for novice and expert players regarding aggressive effects of violent video game play.

From the methodological perspective, the current study presents implications with regard to assessing concretelevel identities. Our initial intention was to develop self-reporting items for measuring the concreteness level, similar to the behavioral identification form (BIF), a frequently-used scale developed by Vallacher and Wegner (1989). BIF items give respondents an action (e.g., "reading) with two answer options describing an action at a concrete (e.g., "following lines of print") and abstract level (e.g., "gaining knowledge"), respectively; and respondents choose an option that is a better description. In the current study, we used Likert-type items, instead of multiple-choice ones, recognizing that concreteness of action identities is constructed on a continuous dimension (Burgoon et al., 2013). Thus, participants were asked to rate how well each concrete-identity item describes their video game experience.

We propose that the failure of revealing the effect of interactivity on concrete-level identities is partly due to the nature of the cognitive process in answering the Likert-type items. That is, questions asking participants to rate their experience on concrete action-identity items may cause them to reflect on, rather than report spontaneously, their cognitive representations. In the current study, the non-interactivity group may have rated high in the Likerttype concrete action-identity items, despite their original action identities being developed at an abstract level. Note that the non-interactivity group observed the character controlled in the video clip, processing the low-level visual information of his actions. Thus, when asked to rate how well concrete identities describe their experience, the non-interactivity group should have reported affirmative responses to the items.

Note that the issue of processing low-level action identities for the non-interactivity group has not emerged in social cognitive studies. The level of concreteness-abstraction in this line of literature is often manipulated by an imagination task, in which, for example, participants are asked to think about a target (e.g., eating breakfast) in terms of overarching end states (i.e., the abstract condition) or specific means (i.e., the concrete condition, Strack, Schwarz, \& Gschneidinger, 1985). Thus, in this imagination-task manipulation, the abstract-condition group hardly processes any concrete identities of a target, barely reporting concrete identities when they answer to the Likerttype concrete identity items.

In addition to the Likert-type items, we employed a thought-listing technique, in which participants were asked to describe their in-game experiences. We expected that the way they do so would reveal the concreteness level of their action identities. We qualified the level of concreteness of participants' responses by applying LCM (Semin \& 
Fiedler, 1991), and results lent support for our prediction on the effect of interactivity on the concrete actionidentities. While LCM has been developed and used to analyze languages used in an interpersonal context (Semin, 2008), recent studies employ the model to analyze individuals' cognitive structure on self-knowledge (e.g., personal memory, Stephan, Sedikides, \& Wildschut, 2012). Based on this finding and existing studies, we propose the superiority of the thought-listing item over the Likert-type measure in assessing concrete action-identity developed from video game play.

Also, results from this study have practical applications concerning how to harness media technologies to influence users. Based on our findings, industry professionals who attempt to persuade audiences via immersive media should consider reinforcing interactivity in their media. For example, an advertisement or public service announcement can harness interactivity in its contents to allow people to experience a product or issue at a concrete level and cause them to be open to persuasive messages. Further, our current study's findings may help explain the advantages of video games as persuasive media (e.g., serious games) over traditional non-interactive ones (e.g., Peng, 2008). Interactivity features of video games would allow players to experience video game contents at a concrete level by engaging them in specific actions, thus render them susceptible to a persuasive message.

\section{Limitations and Directions for Future Research}

Findings of the current study should be understood under several limitations. Particularly, the effect of interactivity presented in the current study was not of a strong effect-size. Note that our manipulation of interactivity and noninteractivity did not generate a significant total effect, while our analysis for the mediational effect evidenced the existence of the indirect effect of interactivity. This result indicates that interactivity induces the persuasive effect only when it generates concrete-level action identities. Given the effect of interactivity is moderate and conditional, future studies should be conducted to replicate the findings of the current study to strengthen the evidence of the persuasive effect of interactivity and the mediational role of concrete-level action identities.

In addition, it is important to point out that our findings are based on the specific video game, employed in the particular experimental setting, calling for further examination to generalize findings beyond the medium and procedure used in the present study. We selected the online hunting simulation game for the desirable conditions required in the current study, such as realistic visual representations and a high degree of freedom in controls of specific actions of a character. One may conjecture that our current study's findings are valid only for the hunting game used in the present study. As hunting is not the most preferred activity for young adults, our study participants might have felt uncomfortable with detailed bodily actions involved in hunting and might have found the video game embarrassing to play. Because of possible discomfort at the contents of the video game, participants might have paid greater attention to concrete level identities, thus being susceptible to a new meaning of hunting. Accordingly, future research should test effects of media interactivity using different video games, particularly contents with which participants feel comfortable, and evidence that interactivity may generate the persuasive effect even for video games with pleasant contents. Especially encouraged is the testing of media possessing broader practical implications (e.g., media promoting desirable outcomes in health-related or educational domains), as these often seek to deliver persuasive messages to induce behavioral changes in their users.

Also, the specific procedure of the current experiment would influence our results, so future studies would attempt to replicate the findings of the current study using different experimental settings. Given that individuals' mental representations can be influenced by an experimental procedure in a subtle way (Bargh \& Chartrand, 2000), the order of items presented in a questionnaire may influence the concreteness level of actions participants construct. In the current experiment, participants evaluated the news article before completing the post-game questionnaire. This procedure exposed participants to specific descriptions of hunting in the news article, then to the concrete action-identities of hunting used in the Likert-type items. While applying the same experimental procedure to all participants guarantees the internal validity of the experiment, results may not be same in different experimental settings. Thus, future studies would secure the generalizability of the findings by using different experimental settings. 
Further, future research may generalize our findings by employing media with greater ecological validity. For the non-interactive condition, we used pre-recorded game-play to control for factors other than interactivity (e.g., video game contents). As a result, the interactivity and the non-interactivity groups were exposed to similar media contents except that the interactivity group controlled the character, while the non-interactivity group viewed the similar character's behaviors. However, participants in the non-interactive condition may have felt bored watching the pre-recorded video of the other's game play, not fully developing high-level action-identities from their mediause experience. We expect that when participants use a non-interactive medium with an interesting story, they are likelier to interpret meanings of the story. As watching the other's game play is not a common way to consume non-interactive media, future research can expand in this regard by comparing an interactive medium to an existing non-interactive medium, such as an animation with a meaningful story. Moreover, bored participants of the non-interactivity group would not have paid attention to the news article they were asked to read, thus revealed less agreement to a new meaning of hunting. Accordingly, future research may control for the level of boredom in media use and level of attention to a persuasive message, to rule out the alternative explanation.

Another generalizability-related limitation of the current study is from the fact that the participants were inexperienced with the video game. Thus, one may suspect that the findings of the interactivity effect would be valid only for people without prior experience in video-game contents. In line with this suspicion, recent studies showed that the more experienced people are with a video game, the likelier they are to develop abstract identities from their video game experience (Ewell et al., 2018; Matthews, 2015). This finding is in line with the action identification theory (Vallacher \& Wegner, 2012), which posits that abstract identities often are utilized to construe actions people can perform fluently. Also, the participants recruited in the current study were not representative regarding their demographic characteristics. In particular, the gender of the participants was not balanced across the experimental conditions and their ages were limited to a certain age group (see Table 1). Thus, future studies would test the generalizability of the interactivity effect for participants with different game experience and demographic characteristics: Whether playing a video game, compared to watching someone playing, would generate an effect strong enough to cause experienced and inexperienced players with balanced demographic characteristics to develop concrete action identities and render them vulnerable to a new idea of actions they performed in the video game.

More, for greater rigor in methodology, future studies may use an experimental design with greater statistical power by employing a yoked control design (Wasserman, 2018). In the current study, all participants in the noninteractive condition watched the same media contents, whereas participants in the interactive condition were exposed to different media contents as the interactivity group were able to control the contents. This experiment design reflects the realistic setting of a non-interactive medium being produced and consumed in a single version, and an interactive medium providing users a substantial degree of freedom. Future studies may control these unbalanced sources of variations in a yoked design, by recording a play of a participants in the interactivity group and using the video for a yoked participant in the non-interactivity group. This yoked design allows controlling for unintended bias across interactive and non-interactive conditions, and would result in an increased statistical power for examining interactivity effects.

Also, the current study did not control for other mediators regulating potential underlying mechanisms of immersive media persuasive effects (e.g., enjoyment, Green, Brock, \& Kaufman, 2004; perceived realism, Guadagno, Blascovich, Bailenson, \& McCall, 2007; and presence, Skalski \& Tamborini, 2007). Given the modest effect size of our mediator, including other mediators may override the mediating role of concrete actionidentities. Thus, future research may examine the unique contribution of the concrete action-identities to explain the effect of interactivity by employing potential mediators as covariates. This type of study can provide meaningful implications on what aspects of interactive media should be emphasized to make a medium more persuasive.

Last, future studies can go beyond the concept of interactivity and examine effects of various media technologies using interactivity (e.g., a motion-based controller) or rich sensory information (e.g., 3D graphic or head-mounted display). Because these technologies have the potential to facilitate media users developing concrete-level mental representations, media employing such technologies may render media users susceptible to new ideas on the subject matter of the media. Using the level of concreteness as a mediator, future studies can provide empirical evidence for the effects of various media technologies and the underlying cognitive mechanism of the effects. 


\section{Conclusion}

The current study evidenced the utility of the concrete action identity, an unexplored construct in media-effect research, in understanding how interactive media generate a greater influence than do non-interactive media. We believe that using this social-cognitive construct allows us to apply the novel theoretical framework of mental representation to media-effect research and to reshape our prior understanding of how interactivity influences users' beliefs, extending our body of knowledge on the persuasive effect of interactivity. Considering the limitations of the study, however, our current study's findings should be regarded cautiously, and future studies should continue examining how mental representation can be utilized to media-effect research and whether the current study's findings are replicable in different settings, to build a more solid foundation of the social cognitive perspective.

\section{Acknowledgement}

This work was supported by the National Research Foundation of Korea Grant funded by the Korean Government (NRF-2017S1A3A2066149).

\section{References}

Aarts, H., Gollwitzer, P. M., \& Hassin, R. R. (2004). Goal contagion: Perceiving is for pursuing. Journal of Personality and Social Psychology, 87, 23-37. http://doi.org/10.1037/0022-3514.87.1.23

Anderson, C. A. (2004). An update on the effects of playing violent video games. Journal of Adolescence, 27, 113122. https://doi.org/10.1016/j.adolescence.2003.10.009

Avalanche Studios. (2000). The Hunter [Video game]. Available from: http://www.thehunter.com/

Bailenson, J. N., Patel, K., Nielsen, A., Bajscy, R., Jung, S.-H., \& Kurillo, G. (2008). The effect of interactivity on learning physical actions in virtual reality. Media Psychology, 11, 354-376.

http://doi.org/10.1080/15213260802285214

Banaji, M. R., Lemm, K. M., \& Carpenter, S. J. (2001). The social unconscious. In A. Tesser \& N. Schwarz (Eds.), Blackwell handbook of social psychology: Intraindividual processes (pp. 134-158). Malden, MA, US: Blackwell Publishers Inc.

Bargh, J. A., \& Chartrand, T. L. (2000). The mind in the middle: A practical guide to priming and automaticity research. In H. T. Reis \& C. M. Judd (Eds.), Handbook of research methods in social and personality psychology (pp. 253-285). New York, NY, US: Cambridge University Press.

Bartholow, B. D., Sestir, M. A., \& Davis, E. B. (2005). Correlates and consequences of exposure to video game violence: Hostile personality, empathy, and aggressive behavior. Personality and Social Psychology Bulletin, 31 , 1573-1586. https://doi.org/10.1177/0146167205277205

Blakemore, S.-J., \& Frith, C. (2003). Self-awareness and action. Current Opinion in Neurobiology, 13, 219-224. http://doi.org/10.1016/S0959-4388(03)00043-6

Burgoon, E. M., Henderson, M. D., \& Markman, A. B. (2013). There are many ways to see the forest for the trees: A tour guide for abstraction. Perspectives on Psychological Science, 8, 501-520.

http://doi.org/10.1177/1745691613497964

Carlston, D. E., \& Smith, E. R. (1996). Principles of mental representation. In E. T. Higgins \& A. W. Kruglanski (Eds.), Social psychology: Handbook of basic principles (pp. 184-210). New York, NY, US: Guilford Press. 
Costabile, K. A., \& Klein, S. B. (2008). Understanding and predicting social events: The effects of narrative construction on inference generation. Social Cognition, 26, 420-437. http://doi.org/10.1521/soco.2008.26.4.420

Ewell, P. J., Hamilton, J. C., \& Guadagno, R. E. (2018). How do videogame players identify their actions? Integrating Action Identification Theory and videogame play via the Behavior Identification Form-Gamer. Computers in Human Behavior, 81, 189-197. https://doi.org/10.1016/j.chb.2017.12.019

Fazio, R. H., \& Olson, M. A. (2003). Implicit measures in social cognition research: Their meaning and use. Annual Review of Psychology, 54, 297-327. http://doi.org/10.1146/annurev.psych.54.101601.145225

Förster, J., \& Liberman, N. (2007). Knowledge activation. In A. W. Kruglanski \& E. T. Higgins (Eds.), Social psychology: Handbook of basic principles (2nd ed., pp. 201-231). New York, NY, US: Guilford Press.

Green, M. C., Brock, T. C., \& Kaufman, G. F. (2004). Understanding media enjoyment: The role of transportation into narrative worlds. Communication Theory, 14, 311-327. http://doi.org/10.1111/j.1468-2885.2004.tb00317.x

Guadagno, R. E., Blascovich, J., Bailenson, J. N., \& McCall, C. (2007). Virtual humans and persuasion: The effects of agency and behavioral realism. Media Psychology, 10, 1-22. Retrieved from

https://www.tandfonline.com/doi/full/10.1080/15213260701300865

Hayes, A. F. (2009). Beyond Baron and Kenny: Statistical mediation analysis in the new millennium. Communication Monographs, 76, 408-420. http://doi.org/10.1080/03637750903310360

Heider, F., \& Simmel, M. (1944). An experimental study of apparent behavior. American Journal of Psychology, 57, 243-259. http://doi.org/10.2307/1416950

Klimmt, C., Hefner, D., \& Vorderer, P. (2009). The video game experience as "true" identification: A theory of enjoyable alterations of players' self-perception. Communication Theory, 19, 351-373.

http://doi.org/10.1111/j.1468-2885.2009.01347.x

Landis, J. R., \& Koch, G. G. (1977). The measurement of observer agreement for categorical data. Biometrics, 33, 159-174. http://doi.org/10.2307/2529310

Ledgerwood, A., Trope, Y., \& Chaiken, S. (2010). Flexibility now, consistency later: Psychological distance and construal shape evaluative responding. Journal of Personality and Social Psychology, 99, 32-51.

http://doi.org/10.1037/a0019843

Lin, J.-H. (2013). Identification matters: A moderated mediation model of media interactivity, character identification, and video game violence on aggression. Journal of Communication, 63, 682-702.

http://doi.org/10.1111/jcom.12044

Matthews, N. L. (2015). Too good to care: The effect of skill on hostility and aggression following violent video game play. Computers in Human Behavior, 48, 219-225. https://doi.org/10.1016/j.chb.2015.01.059

Oh, J., \& Sundar, S. S. (2015). How does interactivity persuade? An experimental test of interactivity on cognitive absorption, elaboration, and attitudes. Journal of Communication, 65, 213-236. http://doi.org/10.1111/jcom.12147

Peng, W. (2008). The mediational role of identification in the relationship between experience mode and selfefficacy: Enactive role-playing versus passive observation. CyberPsychology \& Behavior, 11, 649-652. http://doi.org/10.1089/cpb.2007.0229

Petty, R. E., \& Briñol, P. (2012). The elaboration likelihood model. In P. A. M. van Lange, A. Kruglanski, \& E. T. Higgins, Handbook of theories of social psychology (Vol. 1, pp. 224-245). London, UK: Sage. 
Rafaeli, S., \& Ariel, Y. (2007). Assessing interactivity in computer-mediated research. In A. N. Joinson, K. Y. A. McKenna, T. Postmes, \& U.-D. Reips (Eds.), Oxford handbook of Internet psychology (pp. 71-88). Oxford, UK: Oxford University Press.

Roskos-Ewoldsen, D. R., Roskos-Ewoldsen, B., \& Carpentier, F. D. (2009). Media priming: An updated synthesis. In J. Bryant \& M. B. Oliver (Eds.), Media effects: Advances in theory and research (3rd ed., pp. 74-93). New York, NY, US: Routledge/Taylor \& Francis.

Sanchez-Vives, M. V., \& Slater, M. (2005). From presence to consciousness through virtual reality. Nature Reviews Neuroscience, 6, 332-339. http://doi.org/10.1038/nrn1651

Schultheis, M. T., \& Rizzo, A. A. (2001). The application of virtual reality technology in rehabilitation. Rehabilitation Psychology, 46, 296-311. http://doi.org/10.1037/0090-5550.46.3.296

Semin, G. R. (2008). Language puzzles: A prospective retrospective on the linguistic category model. Journal of Language and Social Psychology, 27, 197-209. https://doi.org/10.1177/0261927X07313664

Semin, G. R., \& Fiedler, K. (1988). The cognitive functions of linguistic categories in describing persons: Social cognition and language. Journal of Personality and Social Psychology, 54, 558-568. https://doi.org/10.1037/00223514.54.4.558

Semin, G. R., \& Fiedler, K. (1989). Relocating attributional phenomena within a language-cognition interface: The case of actors' and observers' perspectives. European Journal of Social Psychology, 19, 491-508.

https://doi.org/10.1002/ejsp.2420190602

Semin, G. R., \& Fiedler, K. (1991). The linguistic category model, its bases, applications and range. European Review of Social Psychology, 2, 1-30. https://doi.org/10.1080/14792779143000006

Shrum, L. J. (2009). Media consumption and perceptions of social reality: Effects and underlying processes. In J. Bryant \& M. B. Oliver (Eds.), Media effects: Advances in theory and research (3rd ed., pp. 50-73). New York, NY, US: Routledge/Taylor \& Francis.

Skalski, P., \& Tamborini, R. (2007). The role of social presence in interactive agent-based persuasion. Media Psychology, 10, 385-413. http://doi.org/10.1080/15213260701533102

Slater, M. D., Rouner, D., \& Long, M. (2006). Television dramas and support for controversial public policies: Effects and mechanisms. Journal of Communication, 56, 235-252. https://doi.org/10.1111/j.1460-

2466.2006.00017.x

Stephan, E., Sedikides, C., \& Wildschut, T. (2012). Mental travel into the past: Differentiating recollections of nostalgic, ordinary, and positive events. European Journal of Social Psychology, 42, 290-298.

https://doi.org/10.1002/ejsp.1865

Steuer, J. (1992). Defining virtual reality: Dimensions determining telepresence. Journal of Communication, 42(4), 73-93. http://doi.org/10.1111/j.1460-2466.1992.tb00812.x

Strack, F., Schwarz, N., \& Gschneidinger, E. (1985). Happiness and reminiscing: The role of time perspective, affect, and mode of thinking. Journal of Personality and Social Psychology, 49, 1460-1469.

https://doi.org/10.1037/0022-3514.49.6.1460

Sundar, S. S. (2004). Theorizing interactivity's effects. The Information Society, 20, 385-389. http://doi.org/10.1080/01972240490508072

Tingley, D., Yamamoto, T., Hirose, K., Keele, L., \& Imai, K. (2014). mediation: R package for causal mediation analysis. Journal of Statistical Software, 59(5). http://dx.doi.org/10.18637/jss.v059.i05 
Trope, Y., \& Liberman, N. (2010). Construal-level theory of psychological distance. Psychological Review, 117, 440463. http://doi.org/10.1037/a0018963

Uleman, J. S., Newman, L. S., \& Moskowitz, G. B. (1996). People as flexible interpreters: Evidence and issues from spontaneous trait inference. In M. P. Zanna (Ed.), Advances in experimental social psychology (Vol. 28, pp. 211-279). San Diego, CA, US: Academic Press.

Vallacher, R. R., \& Wegner, D. M. (1989). Levels of personal agency: Individual variation in action identification. Journal of Personality and Social Psychology, 57, 660-671. http://doi.org/10.1037/0022-3514.57.4.660

Vallacher, R. R., \& Wegner, D. M. (2012). Action identification theory. In P. A. M. van Lange, A. W. Kruglanski, \& E. T. Higgins (Eds.), Handbook of theories in social psychology (Vol. 1, pp. 327-348). London, UK: Sage.

van der Weiden, A., Aarts, H., \& Ruys, K. (2013). On the nature of experiencing self-agency: The role of goals and primes in inferring oneself as the cause of behavior. Social and Personality Psychology Compass, 7, 888-904. http://doi.org/10.1111/spc3.12075

Wasserman, E. A. (2018). Yoked control procedure. In N. Salkind (Ed.), Encyclopedia of research design (Vol. 3). Retrieved from https://doi.org/10.4135/9781412961288

Wegner, D. M., Vallacher, R. R., Macomber, G., Wood, R., \& Arps, K. (1984). The emergence of action. Journal of Personality and Social Psychology, 46, 269-279. http://doi.org/10.1037/0022-3514.46.2.269

Westerman, D., Spence, P. R., \& Lin, X. (2015). Telepresence and exemplification in health messages: The relationships among spatial and social presence and exemplars and exemplification effects. Communication Reports, 28, 92-102. https://doi.org/10.1080/08934215.2014.971838

Wu, G. (2005). The mediating role of perceived interactivity in the effect of actual interactivity on attitude toward the website. Journal of Interactive Advertising, 5(2), 29-39. http://doi.org/10.1080/15252019.2005.10722099

Zillmann, D. (2006). Exemplification effects in the promotion of safety and health. Journal of Communication, 56(Suppl. 1), S221-S237. https://doi.org/10.1111/j.1460-2466.2006.00291.x

Zillmann, D., Gibson, R., Sundar, S. S., \& Perkins, J. W. Jr. (1996). Effects of exemplification in news reports on the perception of social issues. Journalism \& Mass Communication Quarterly, 73, 427-444.

https://doi.org/10.1177/107769909607300213

\section{Appendix A}

\section{Action Identity Items}

\section{How-to-hunt items.}

Carrying a rifle with ammunition

Slowing down breathing*

Looking around for a target

Waiting for a target under cover

Dressing in camouflage*

Loading your rifle*

Aiming at the target

Walking quietly through the woods*

Firing your rifle* 
Why-to-hunt items.

Empowering oneself in the wilderness*

Exerting influence over nature

Exercising a cultural practice

Being at one with nature*

Engaging in an outdoor activity*

Revealing the wild nature of human beings

Utilizing natural resources*

Exploring the wilderness*

Managing ecosystems*

Scale: Very poorly 1-------2------3------4------5 Very well

Note. Asterisks indicate the items selected for the concrete- and abstract-identity scores.

\section{Agreement items.}

The news article is convincing.

I support the ideas in the news article.

The arguments in the news article are valid.

The results of Dr. Johnson's study seemed to be trustworthy.

I think Dr. Johnson's findings are true for people in other states.

I expect the effects of deer hunting will be about the same to me.

Scale: Strongly disagree 1-------2------3------4------5-----6------7 Strongly agree

\section{Appendix B: News Articles}

\section{Hidden Dangers for Hunters, Study Finds}

The great orange army, comprised of more than 500,000 hunters, are eagerly preparing for the opening of another firearms deer hunting season in Michigan. Many bag a deer, but along with success comes a hidden risk. Researchers from Michigan's Beaumont Hospital have identified the danger of a heart attack among men with heart disease.

Susan Johnson and colleagues from Beaumont Hospital's Cardiac Rehabilitation program were the first to conduct a study on the risk of heart attack from deer hunting. The results were published in August 2013 in The American Journal of Cardiology. Researchers sent 25 hunters into the woods equipped with heart rate monitors. They found that many hunting activities increased the men's heart rates soaring to peak heart levels achieved during cardiac stress testing.

"The heart rate of most hunters would almost double upon seeing a deer, even though they were standing totally still," says Johnson an exercise physiologist. "After shooting, gutting and dragging a deer out of the woods the hunters' heart rates were well above the maximum heart rate. Hunting is a strenuous activity and can trigger heart disease in a sedentary male. Exceeding the maximum heart rate for a sustained period places a hunter at risk for heart attack," adds Johnson. "The hunters in our study were often unaware of what was happening to their hearts during the hunt."

\section{Hidden Benefits for Hunters, Study Finds}

The great orange army, comprised of more than 500,000 hunters, are eagerly preparing for the opening of another firearms deer hunting season in Michigan. Many bag a deer, and along with success comes a hidden benefit. 
Researchers from Michigan's Beaumont Hospital have identified the cognitive benefits of hunting among men with cognitive disorder.

Susan Johnson and colleagues from Beaumont Hospital's Psychiatric Rehabilitation program were the first to conduct a study on the psycho-cognitive benefits of deer hunting. The results were published in August 2013 in The American Journal of Cognition. Researchers sent 25 hunters into the woods equipped with brain activity monitors. They found that many hunting activities increased the men's cognitive activity reaching maximum levels achieved during cognitive training testing.

"The cognitive activity of the most hunters would almost double upon seeing a deer, because it activates procedural knowledge of hunting," says Johnson a cognitive physiologist. "While shooting, chasing and aiming a rifle at a deer in the woods the hunters' cognitive functions were constantly active. Hunting is a strategic activity and can be a great cognitive exercise for an elderly male. Keeping cognitive function active for a sustained period brings brain health to a hunter," adds Johnson. "The hunters in our study were often unaware of what was happening to their brain during the hunt."

\section{Appendix C: Coding Categories}

Descriptive Action Verb (DAV): Graphical action or motion

- Characteristic Features: Reference to a specific and visible behavioral event; Objective description of observable events

- Classification criteria: Reference to one particular activity and to a physically invariant feature of the action

- Example: walking, shooting, aiming

Interpretive Action Verb (IAV): Behavior that can be done by more than one action

- Characteristic Features: Reference to a not directly visible single behavioral event; Interpretation beyond description

- Classification criteria: Reference to a multitude of behaviors; Defined action with beginning and end; Positive and negative semantic valence

- Example: hunting, tracking, killing, stalking

State action verb (SAV): Mental state as a result of specific behavior

- Characteristics features: See interpretative action verb, but no reference to concrete situation frames; Reference to states evoked in object of sentence by unspecified action

- Classification criteria: As interpretative action, except the verb expresses emotional consequence of action rather than referring to action as such

- Example: Surprise, amaze, anger, anticipated, focus, patience, aware

\section{State verb (SV): Emotional state; No obvious behavior related}

- Characteristics features: Enduring states, Reference to a social object, but not to a situation; No context reference; Interpretation beyond mere description

- Classification criteria: Reference to mental and emotional state; No clear definition of beginning and end; Do not readily take progressive forms

- Example: (feel) scary, exited, anxious, guilt, admire, hate, appreciate

Adjective (ADJ): Describing the state; what is the object like

- Characteristics features: Highly abstract person description; No object or situation reference; No context reference; Highly interpretative; detached from specific behaviors 
- Classification criteria: Characteristics of a person; Qualification of object or act

- Example: (be) similar, serene, realistic, exciting

\section{Correspondence to:}

Young June Sah

Kangwon National University

1 Kangwondaehak-gil

Chuncheon-si, Gangwon-do, 24341

South Korea

Email: sahyoungjune(at)gmail.com

Editorial record: First submission received on January 12, 2019. Revisions received on August 13, 2019, September 27, 2019 and October 15, 2019. Accepted for publication on October 16, 2019.

Editor in charge: David Smahel

\section{About Authors}

Young June Sah (PhD, Michigan State University) is a Research Professor in the Social Integration Research Center at Kangwon National University. His research interests include psychological and behavioral effects of media technologies and their cognitive mechanisms.

Wei Peng (PhD, University of Southern California) is a Professor in the Department of Media and Information, Michigan State University. Her research focuses on the psychological and social mechanisms of behavior change and their application in the design of interactive media for health and wellness promotion. 